
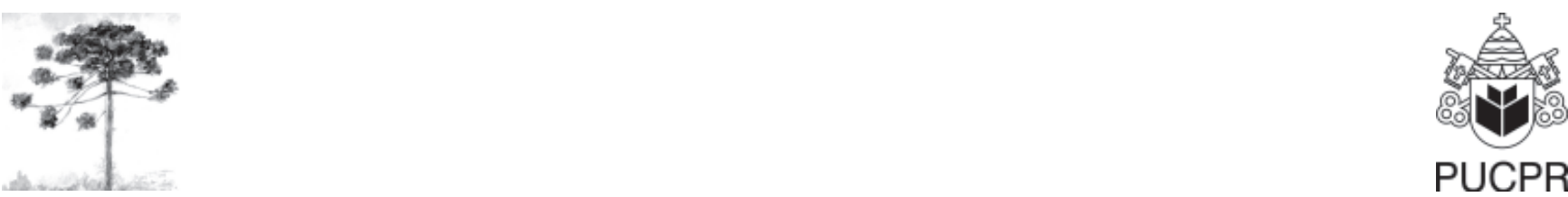

\title{
OCORRÊNCIA DE ENDOPARASITAS EM AMOSTRAS DE FEZES DE CÃES (Canis familiaris) DA REGIÃO METROPOLITANA DE CURITIBA, PARANÁ - BRASIL
}

\author{
Ocurrence of endoparasites in fecal samples from dogs (Canis familiaris) \\ of Curitiba - Paraná - Brazil, metropolitan region
}

\section{Luiz Carlos Leite ${ }^{[1]}$, Silvana Maris Círio ${ }^{[2]}$, Mário Antonio Navarro-Silva ${ }^{[3]}$, Ana Carolina Bazo Zadorosnei ${ }^{[4]}$, Enio Luz ${ }^{[5]}$, Leide Parolin Marinoni ${ }^{[6]}$, Selene Círio Leite ${ }^{[7]}$, Diego Lunelli ${ }^{[8]}$}

\footnotetext{
${ }^{[1]}$ Professor Doutor colaborador do Curso de Medicina Veterinária da Universidade Estadual do Centro-Oeste (Unicentro), Guarapuava, PR - Brasil, e-mail: tudolica@pop.com.br

${ }^{[2]}$ Professora Doutora titular do Curso de Medicina Veterinária da Pontifícia Universidade Católica do Paraná (PUCPR), Curitiba, PR - Brasil.

${ }^{[3]}$ Professor Doutor do Departamento de Entomologia da Universidade Federal do Paraná (UFPR), Curitiba, PR - Brasil.

${ }^{[4]}$ Médica Veterinária, Curitiba, PR - Brasil.

${ }^{[5]}$ Professor titular, Departamento de Patologia Básica da Universidade Federal do Paraná (UFPR), Curitiba, PR - Brasil.

[6] Professora do Departamento de Pediatria do Hospital das Clínicas (UFPR), Curitiba, PR - Brasil

${ }^{[7]}$ Médica Veterinária, Mestranda em Entomologia pela Universidade Federal do Paraná (UFPR), Curitiba, PR - Brasil.

${ }^{[8]}$ Médico Veterinário, Professor colaborador do Curso de Medicina Veterinária da Universidade do Contestado (UnC), Canoinhas, SC - Brasil, Mestrando em Ciência Animal pela Pontifícia Universidade Católica do Paraná (PUCPR), Curitiba, PR - Brasil.
}

\section{Resumo}

Amostras de fezes de 387 cães sem distinção de faixa etária, sexo ou raça e oriundos de diferentes locais da região metropolitana de Curitiba, PR, foram coletadas para diagnóstico laboratorial mediante exames coproparasitológicos realizados no laboratório de Parasitologia Veterinária da Pontifícia Universidade Católica do Paraná (PUCPR) em São José dos Pinhais, PR, entre os meses de fevereiro de 2000 a dezembro de 2005. As fezes foram colhidas individualmente e processadas pelo método de Hoffman, Pons \& Janer (1934) (1). Os resultados obtidos comprovaram contaminação por ovos de Ancylostoma spp. (18,6\%), Trichuris vulpis (10,35\%), Toxocara spp. (1,29\%), Dipylidium caninum (0,77\%), Cystoisospora sp. (0,51\%), Candida spp. (0,51\%) e Giardia spp. $(0,25 \%)$. O elevado índice de cães portadores de infecção por endoparasitas 
evidencia o risco potencial de transmissão de zoonoses a que está exposto o ser humano que convive com animais infectados. Muitas vezes um animal aparentemente sadio está eliminando no solo fezes contendo ovos embrionados desses parasitas. As crianças podem facilmente infectar-se ou reinfectar-se pela ingestão de terra contaminada.

Palavras-chave: Cães; Ancylostoma; Toxocara; Parasitas gastrintestinais.

\begin{abstract}
Fecal samples from 387 dogs without distinction of age, sex or race from different locations in the metropolitan region of Curitiba, Parana State, were collected for laboratory coproparasitologic diagnosis, conducted at the Laboratory of Veterinary Parasitology from Pontificia Universidade Catolica do Paraná (PUCPR) in São José dos Pinhais, Paraná, collected from February 2000 to December 2005. The fecal samples were collected individually and processed by the method of Hoffman, Pons E Janer (1934) (1). The results showed contamination by Ancylostoma spp. (18,6\%), Trichuris vulpis (3,1\%), Toxocara spp. $(1,29 \%)$, Dipylidium caninum $(0,77 \%)$, Cystoisospora $s p .(0,51 \%)$, Candida spp. $(0,51 \%)$ and Giardia spp. $(0,25 \%)$. The high rate of dogs with endoparasites infection show the potencial risk of transmition of zoonosis to human that live with infected dogs. Many times, this animal looks healthy, but expel on soil embryonated eggs with his feces, and children are the most likely to become infected or reinfected by eating dirt soil.
\end{abstract}

Keywords: Dogs; Ancylostoma; Toxocara; Gastointestinal parasites.

\section{INTRODUÇÃO}

Em saúde pública e clínica veterinária, o estudo da epidemiologia das doenças parasitárias de pequenos e grandes animais é fundamental, uma vez que vários gêneros de helmintos e protozoários, dotados de considerável potencial zoonótico, habitualmente utilizam-se dos animais de companhia como hospedeiros e podem contaminar o homem com relativa facilidade. Parasitas intestinais podem desenvolver afecções responsáveis por alta morbidade e mortalidade em cães jovens e adultos. O fácil acesso a praças e parques, a falta de bom senso de proprietários que não recolhem as fezes de seus animais de estimação, deixando-as expostas em vias públicas e locais de lazer, somados à carência de informações a respeito dos mecanismos de transmissão das doenças parasitárias contribuem sobremaneira na contaminação do ambiente. As crianças e adultos podem infectar-se por ingestão acidental de ovos larvados e cistos presentes no solo (geofagia), em hortaliças consumidas cruas (2), fômites, mãos ou ainda por migração cutânea de helmintos em estágio larval infectante, oriundos das fezes de animais contaminados. Os ascarídeos Toxocara canis, Toxascaris leonina, Toxocara cati, parasitas habituais de cães e gatos, podem provocar infecção acidental em seres humanos mediante ingestão de ovos embrionados, cujas larvas (Larva Migrans Visceral) liberadas no organismo, sobrevivem vários meses e provocam lesões irreversíveis em sua migração somática por via linfática ou circulação portal, atingindo diversos órgãos (3-9). O hospedeiro humano também pode infectar-se ingerindo fígado cru de aves ou mamíferos, principalmente bovinos, contendo larvas encapsuladas de Toxocara canis $(6,10)$. Quando as larvas atingem o globo ocular dão origem a uma patologia denominada Larva Migrans Ocular (1113). A sensibilidade do paciente, o órgão afetado e o número de larvas invasoras são fatores importantes na determinação do grau de infecção. O contato direto com cães e gatos parasitados tem pouco destaque na transmissão da doença, visto que os ovos necessitam de um período de incubação no meio ambiente para se tornarem infectantes. A presença de formas adultas de Toxocara cati infectando crianças foi reportada por Eberhard e Alfano (14) e Talaizadeh et al. (5). 
A infecção humana por Trichuris vulpis é muito rara. A respeito desse aspecto do problema conhecem-se poucos relatos nas últimas quatro décadas $(15,16)$. Em crianças e adultos, as formas adultas desse parasita também podem desencadear infecções intestinais, úlceras duodenais acompanhadas por vômitos, náuseas, dores abdominais e diarreia crônica com muco ou sanguinolenta $(17,18)$. Foram descritos dois casos de desenvolvimento atípico de Larva Migrans Visceral em seres humanos provocada por T. vulpis (19).

O cestoda Dipylidium caninum parasita cães e gatos, ocasionalmente podendo infectar crianças (20), causando diarreia, dores abdominais e prurido anal (21-23).

Ancylostoma caninum e Ancylostoma braziliense são estrongilídeos parasitas de cães e gatos. Suas fêmeas eliminam ovos junto com as fezes dos hospedeiros. Posteriormente os ovos embrionam e dispersam-se no ambiente (24). Larvas infectantes (Larva Migrans Cutânea) de $A$. braziliense e $A$. caninum penetram na epiderme de adultos e crianças por contato direto (25). Frequentemente, prurido intenso é observado e o ato de coçar a região afetada pode levarà instalação de infecções bacterianas secundárias. Reação serpiginosa e dermatite são achados comuns nos membros inferiores, pés, nádegas, mãos, face e couro cabeludo (26-28).

Os protozoários de distribuição cosmopolita Giardia spp. e Cystoisospora spp. são importantes agentes infecciosos. Conforme Mendesde-Almeida et al. (29), a espécie mais estudada, Giardia intestinalis (sinonímias: G. duodenalis; G. lamblia) infecta, além de cães e gatos, outras espécies de mamíferos, inclusive seres humanos. Monis et al. (30), utilizando técnicas moleculares de investigação, constataram que a espécie Giardia duodenalis inclui um complexo de oito genótipos principais. O ciclo dos protozoários do gênero Giardia é direto. As formas infectantes são eliminadas juntamente com as fezes e podem permanecer viáveis por vários meses em ambientes úmidos. A transmissão é fecaloral e a infecção de um hospedeiro em potencial pode ocorrer pela ingestão de água, alimento ou fezes contendo cistos esporulados infectantes.

A ocorrência de endoparasitas gastrintestinais tem sido citada no Brasil e em outros países. Na Hungria, Fok et al. (31) examinaram 490 amostras de fezes de cães e obtiveram índices de contaminação igual a $54,40 \%$ para Toxocara canis, 43,70\% por Trichuris vulpi e 21,20\% para Ancylostoma sp. No estado americano do Colorado, Hackett e Lappin (32) analisaram 130 amostras de fezes de cães domésticos e encontraram ovos ou cistos de parasitas em 34 (26,1\%) amostras. Toxocara canis, Ancylostoma caninum e Giardia sp. foram observados em maior proporção. Na cidade de Ica no Peru, Trillo-Altamirano et al. (33) avaliaram 324 amostras fecais de cães, registrando índice geral de positividade igual a 40,12\%. Houve predomínio de infecções simples pelos gêneros Toxocara canis (19,75\%), Ancylostoma caninum (9,26\%), Dipylidium caninum $(8,64 \%)$ e Toxascaris leonina $(6,17 \%)$. $\mathrm{Na}$ cidade de Peniche, Portugal, Crespo et al. (34) obtiveram 66 amostras positivas em 648 amostras estudadas. Em $53(80,30 \%)$ foram identificadas infecções simples, destacando-se Ancylostama spp. em $26(39,40 \%)$ delas. Foi reportada associação parasitária pelos gêneros Taenia spp. / Ancylostomaspp., Ancylostoma spp. / Toxocara spp. e Ancylostoma spp. / Trichuris spp. em 13 amostras (19,70\%).

No Brasil, Fernandes et al. (35), em Curitiba, Estado do Paraná, analisaram prospectivamente 1.065 amostras de fezes de cães, obtendo 797 amostras (74,84\%) com ovos de Toxocara spp., Ancylostoma spp. e Trichuris sp.; Krychak et al. (36), em avaliações de amostras fecais de 172 cães pela técnica de Willis-Mollay, registraram infecção em 122 animais (70,9\%), demonstrando a presença de Ancylostoma spp. em $50(29,06 \%)$, Toxocara spp. em duas $(1,16 \%)$, Dipylidium caninum em duas $(1,16 \%)$ e Cystoisospora spp. em uma amostra $(0,58 \%)$. A mesma pesquisa registrou a infecção mista por Ancylostoma spp. e Trichuris spp. em 31 (18,02\%) e Cystoisospora spp., Ancylostoma spp., Trichuris spp. e Toxocara spp. em sete $(4,06 \%)$ das amostras avaliadas. Ainda em Curitiba, Tesseroli et al. (37) relataram a ocorrência de parasitas intestinais em 280 amostras de fezes examinadas, comprovando a contaminação por Toxocara spp., Trichuris sp., Ancylostoma sp., Giardia sp. e Cystoisospora sp. em 54 (19,28\%), sendo 52 $(18,57 \%)$ infectadas por apenas uma espécie de parasita e $2(0,7 \%)$ com infecções associadas. Em Londrina, Chieffi e Muller (38) obtiveram 70 $(44,3 \%)$ amostras de fezes infectadas por Toxocara canis, de 158 examinadas, e Santos et al. (28) avaliaram amostras fecais colhidas de 150 cães com diarreia aguda, encontrando 44 (29,30\%) infectadas, sendo Giardia sp. (9,30\%), Cystoisospora (7,30\%), Ancylostoma sp. (7,30\%), Toxocara sp. (4,00\%) e Trichuris vulpis (1,30\%). Do total avaliado foram detectadas 32 infecções simples $(72,70 \%)$ e 
12 associações $(27,30 \%$ ). No Estado de São Paulo, Oliveira-Sequeira et al. (39) examinaram amostras de fezes de 271 cães, registrando grau de infecção por Ancylostoma sp. $(23,60 \%)$, Toxocara canis (5,50\%), Trichuris vulpis $(4,80 \%)$, Spirocerca lupi $(1,90 \%)$, Dipylidium caninum $(0,70 \%)$, Giardia sp. $(12,20 \%)$, Cystoisospora sp. (8,50\%) e Sarcocystis sp. $(2,20 \%)$. Em Santa Maria, Rio Grande do Sul, 240 amostras de fezes de cães examinadas por Silva et al. (40) apresentaram alto percentual de infecção por Ancylostoma sp. (69,60\%), sendo também identificados Toxocara sp. (15,00\%), Giardia sp. $(12,20 \%)$, Trichuris sp. (11,25\%), Cryptosporidium sp. $(8,75 \%)$ e Dipylidium caninum $(3,75 \%)$.

O presente estudo teve por finalidade avaliar a ocorrência de protozoários e helmintos intestinais em fezes de cães e gatos da região metropolitana de Curitiba, no período compreendido entre fevereiro de 2000 e dezembro de 2005 .

\section{MATERIAL E MÉTODOS}

Amostras de fezes de 387 cães sem distinção de faixa etária, sexo ou raça foram coletadas individualmente entre fevereiro de 2000 a dezembro de 2005 em diferentes locais da região metropolitana de Curitiba, Paraná, mantidas em refrigeração e encaminhadas ao Laboratório de Parasitologia Veterinária da Pontifícia Universidade Católica do Paraná (PUCPR) em São José dos Pinhais, Paraná, para serem avaliadas mediante exames coproparasitológicos, no máximo em 24 horas após a coleta. A técnica utilizada para realização dos exames foi descrita por Hoffmann, Pons e Janer (1), sendo considerado resultado positivo quando a visualização de um ou mais ovos de nematelmintos, oocistos de protozoários ou cápsulas ovígeras de Dipylidium caninum fosse constatada ao microscópio óptico. O material foi identificado de acordo com suas características morfológicas.

\section{RESULTADOS}

De 387 amostras de fezes pesquisadas, $117(30,23 \%)$ apresentaram resultado positivo para uma ou mais espécies parasitárias, sendo 97 $(25,06 \%)$ com uma única parasitose e $20(5,17 \%)$ com associação de agentes parasitários. Não apresentaram contaminação por endoparasitas 270 $(69,77 \%)$ das amostras avaliadas (Figura 1).

O maior índice encontrado nas amostras contaminadas (117) foi referenteàpresença de Ancylostoma sp. $(62,07 \%)$. Infecções pelos gêneros Trichuris sp. $(10,35 \%)$, Toxocara sp. (4,32\%), Dipylidium caninum (2,58\%), Cystoisospora spp. (1,72\%), Candida sp. (1,72\%) e Giardia spp. $(0,86 \%)$ foram igualmente registradas (Tabela 1). Ainda relatando as amostras contaminadas, em relação às associações parasitárias houve predomínio dos gêneros Ancylostoma spp. / Trichuris spp. (5,17\%), Ancylostomaspp. / Toxocaraspp. (4,32\%) e Ancylostomasp. / Cystoisospora sp. (2,59\%). Infecções múltiplas envolvendo Ancylostoma spp. / Toxocara sp. / Dipylidium caninum, Ancylostoma spp. / Trichuris spp. / Cystoisospora spp. e Ancylostoma spp. / Toxocara spp. / Trichuris spp. / Capillaria spp. / Hymenolepis spp. foram detectadas em menor porcentagem (Tabela 1).

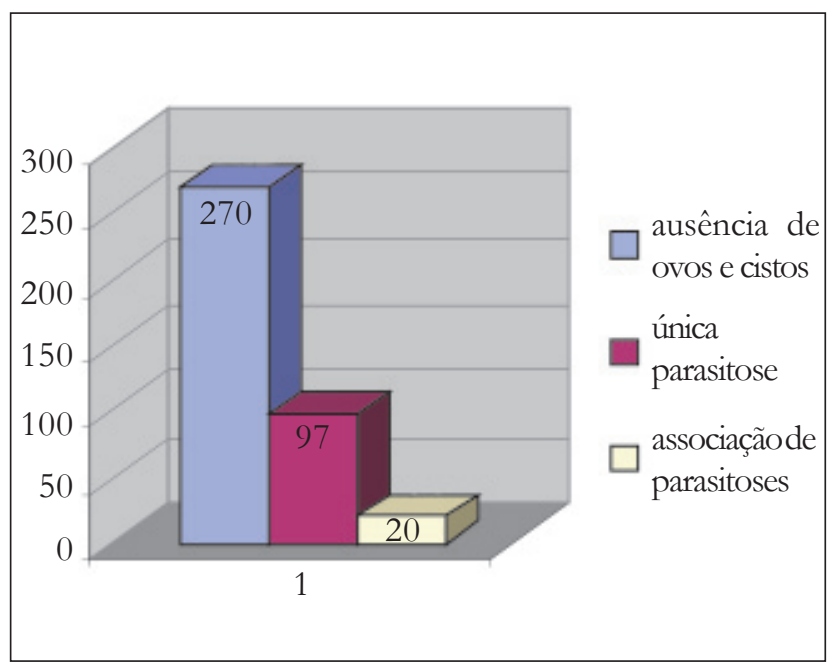

FIGURA 1 - Amostras de fezes analisadas no período de fevereiro de 2000 a dezembro de 2005 
TABELA 1 - Demonstrativo dos índices de exames positivos encontrados em amostras de fezes no período de Fevereiro de 2000 a Dezembro de 2005

\begin{tabular}{lcc}
\hline Endoparasitas & $\begin{array}{l}\text { Amostras } \\
\text { infectadas }\end{array}$ & $\%$ \\
\hline Ancylostoma spp. & 72 & 62,07 \\
Trichuris sp. & 12 & 10,35 \\
Toxocara sp. & 05 & 4,32 \\
Dipylidium caninum & 02 & 1,72 \\
Cystoisospora sp & 02 & 1,72 \\
Candida sp. & 02 & 1,72 \\
Giardia sp. & 01 & 0,86 \\
Ancylostoma sp./ Toxocara sp. & 05 & 4,32 \\
Ancylostoma sp./ Trichuris sp. & 06 & 5,17 \\
Trichuris spp/Candida spp & 01 & 0,86 \\
Ancylostoma sp/ Cystoisospora sp. & 03 & 2,59 \\
Toxocara sp./ Dipylidium caninum & 01 & 0,86 \\
Dipylidium caninum/Cystoisospora & 01 & 0,86 \\
Ancylostoma spp./ Trichuris spp./ Cystoisospora spp & 01 & 0,86 \\
Ancylostoma spp./ Toxocara spp./ Dipylidium caninum. & 01 & 0,86 \\
Ancylostoma spp./ Toxocara spp. / Trichuris.sp./ Capillaria spp / Himenolepis spp. & 01 & 0,86 \\
\hline
\end{tabular}

\section{DISCUSSÃO}

O percentual $(30,23 \%)$ de amostras contaminadas encontradas na presente pesquisa é superior aos achados de Tesseroli et al. (37) em Curitiba, PR (19,28\%), Santos et al. (28) em Londrina, PR $(29,30 \%)$ e igualmente superior aos obtidos por Hackett e Lappin (32) nos EUA (26,10\%) e por Crespo et al. (34) em Portugal (12,32\%). Entretanto, foi inferior aos registros de Fok et al. (31) na Hungria $(54,40 \%)$, Trillo-Altamirano et al. (33) no Peru $(40,12 \%)$ e também aos índices encontrados no Brasil por Fernandes et al. (35) (74,84\%), Krychak et al. (36) em Curitiba, PR (70,90\%), Chieffi e Muller (38) na cidade de Londrina, PR (44,30\%) e Silva et al. (40) em Santa Maria, RS (87,90\%).

No presente estudo, Ancylostoma spp. foi reportado em 18,6\% das amostras avaliadas, taxa inferior à observada na pesquisa de Silva et al. (40) $(69,60 \%)$ na cidade de Santa Maria, RS, e também por Oliveira-Sequeira et al. (39) em São Paulo, que registraram índice de contaminação de $23,60 \%$, em 271 amostras de fezes de cães avaliadas. Cães avaliados por Krychak et al. (36) e Tesseroli et al. (37) na cidade de Curitiba, PR, também apresentaram índices maiores de infecção por Ancylostoma spp. iguais a $29,06 \%$ e $31,45 \%$, respectivamente. Na Hungria, Fok et al. (31) estudaram a ocorrência de endoparasitoses em 490 amostras de fezes de cães, encontrando valores de contaminação por Ancylostoma sp. de 21,20\% e Crespo et al. (34) em Portugal relatam percentual de contaminação igual a 39,40\% em 648 amostras estudadas, sendo que os dois estudos apresentam valores de contaminação maiores que o relatado nesta pesquisa. Santos et al. (28) em Londrina, PR, relataram índice menor de positividade $(7,30 \%)$ para Ancylostoma sp. em 150 amostras de fezes, oriundas de cães com diarreia aguda. Em pesquisa realizada na cidade de Ica, Peru, 162 amostras de fezes caninas avaliadas por Trillo-Altamirano et al. (33) registram também taxas inferiores $(9,26 \%)$ de contaminação por Ancylostoma spp. do total avaliado.

As infecções por Trichuris spp. (10,35\%) apresentaram percentual menor aos encontrados por Fok et al. (31) (43,70\%), de Silva et al. (40) $(69,60 \%)$ e maior dos que os obtidos por Tesseroli et al. (37) (5,60\%). Nos estudos de Santos et al. (28), para esta parasita os índices encontrados foram inferiores aos relatos desta pesquisa $(1,30 \%)$.

As ocorrências de Toxocara spp. (1,29\%) foram inferiores quando comparadas com os registros de Chieffi e Muller (36) (44,30\%) e aos índices encontrados por Silva et al. (40) (15,00\%), aos relatos de Tesseroli et al. (37) e também ao que foi reportado por Santos et al. (28) (4,00\%). A taxa de infecção 
encontrada neste estudo foi superior a demonstrada por Krychak et al. (36) (1,16\%).

Em relação à contaminação por Dipylidium caninum $(0,77 \%)$, o índice foi inferior aos obtidos por Tesseroli et al. (37) (1,85\%), Krychak et al. (36) $(1,16 \%)$ e também menor aos encontrados por Silva et al. (40) $(3,75 \%)$.

A porcentagem de amostras infectadas por Cystoisospora spp. (0,51\%) foi inferior aos resultados obtidos por Krychak etal. (36) (0,58\%), Tesseroli etal. (37) $(38,90 \%)$ e também por Silva et al. (40) (7,08\%).

$\mathrm{Na}$ presente pesquisa foi registrada contaminação de Giardia spp. de 0,25\%, sendo este valor inferior aos achados porSilva etal. (40) (12,08\%).

\section{CONCLUSÃO}

O elevado índice de infecção por endoparasitas nos animais avaliados no presente trabalho evidencia o risco potencial de transmissão de zoonoses a que estão expostas as pessoas que têm contato direto com animais de estimação. Como fator agravante, a debilidade física e sinais clínicos nem sempre são evidentes, pois muitas vezes o animal apresenta-se aparentemente sadio, mesmo parasitado por uma ou várias espécies de parasitas e eliminando formas infectantes.

A identificação e o conhecimento das infecções por parasitas gastrintestinais em cães são importantes para preservar a sanidade animal e prevenir complicações causadas por protozoários, larvas infectantes e formas adultas de helmintos. A avaliação mediante exames coproparasitológicos de rotina, realizada para detectar quais são os animais portadores, associada ao uso racional de anti-helmínticos de amplo espectro e princípios ativos de qualidade, em muito contribuirão para o decréscimo do índice de infecção.

\section{REFERÊNCIAS}

1. Hoffmann WA, Pons JA, Janer JL. The sedimentation concentration method in Schistosomiasis mansoni. Puerto Rico J Publ Health Trop Med. 1934;9(4):283-98.
2. Vollkopf PCP, Lopes MR, Navarro IT. Ocorrência de enteroparasitos em amostras de alfaces (Lactuca sativa) comercializadas em Porto Murtinho - MS. Arq Ciên Vet Zool Unipar. 2006;9(1):37-40.

3. Bächil H, Minet JC, Gratzl O. Cerebral toxocariasis: a possible case of epiletc seizure in children. Child Nerv Syst. 2004;20(7):468-72.

4. Moiyadi, A, Mahadevan A, Anandh B, Shivashankar RS, Chickadasavaiah YT, Shankar SK. Visceral larva migrans presenting as multiple intracranial and intraspinal abscesses. Neuropathol. 2007;27(4):371-4.

5. Talaizadeh AH, Maraghi S, Jelowdar A, Peyvasteh M. Human toxocariasis: a report of 3 cases. Pak J Med Sci. 2007;23(5):782-4.

6. Hoffmeister B, Glaeser S, Flick H, Pornschlegel S, Suttorp N, Bergmann F. Cerebral toxocariasis after consumption of raw duck liver. Am J Trop Med Hyg. 2007;76(3):600-2.

7. Georgiou C, Efstathiades Y, Dimitriou N, Theophanous M, Voros D. Na unusual case of Toxocara canis of the ascending colon. Euro J Gastro Hep. 2007;19(12):1149-53.

8. Perfetti DJC, Moreno PM, Quintero MEA. Contaminación de suelos con huevos de Toxocara spp. (Nematoda, Ascaridida) em parques públicos de la ciudad de Coro, Estado Falcón, Venezuela. Rev Científica FCV-LUZ. 2007;12(2)117-22.

9. Paludo ML, Falavigna DLM, Elefant GR, Gomes ML, Baggio MLM, Amadei LB, et al. Frequency of toxocara infection in children attended by the health public service of Maringá - South Brazil. Rev Inst Med Trop São Paulo. 2007;49(6):343-8.

10. Lim JH, Lee KSV. Eosinophilic infiltration in Korea: Idiopathic? J Kor Rad. 2006;7(1):4-6.

11. Good B, Holland CV. Taylor MRH, Larragy J, Moriarty P, Regan M. Ocular toxocariasis in school children. Clin Infec Dis. 2004;39 (2):173-8. 
12. Fomda BA, Ahmad NN, Tanveer S, Wani SA. Ocular toxocariasis in a child: a case report from Kashmir, north India. Ind J Med Microbiol. 2007;25(4):411-2.

13. Katagiri S, Oliveira-Sequeira TCG. Zoonoses causadas por parasitas intestinais de cães e o problema do diagnóstico. Arq Inst Biol. 2007;74(2):175-84

14. Eberhard ML, Alfano E. Adult Toxocara cati infections in U.S. children: report of four cases. Am J Trop Med Hyg. 1998;59(3):404-6.

15. Kenney M, Everland LK. Infection of man with T. vulpis: The whipworm of dogs. Am J Clin Pat. 1978;69:199-202.

16. Vasquez TO, Martinez BII, Romero CR, Valencia RS, Tay ZJ. Infección mixta por Trichuris thichiura y por Trichuris vulpis. Rev Gastroenterol Peru. 1997;17(3):255-8.

17. Singh S, Samantary JC, Singh N, Verma EC. Trichuris infection in an Indian tribal population. Parasitol. 1993;79(3):457-8.

18. Dunn JJ, Columbus ST, Aldeen WE, Davis M, Carrol KC. Trichuris vulpis recovered from a pacient with chronic diarrhea and five dogs. J Clin Microbiol. 2002;40(7):2703-4.

19. Sakano T, Hamamoto K, Kobayashi Y, Tsuji M, Usui T. Visceral larva migrans caused by Trichuris vulpis. Arch Did Child. 1989;55(8):631-3.

20. Wijesundera MD, Ranaweera RL. Case report of Dipylidium caninum: a pet associated infection. Ceylon Med J. 1989;34(1):27-30.

21. Marinho RP, Campos DMB, Damaceno FA. Dipylidiun caninum (Dipepididae - Cestoda) relato de dois casos humanos. Rev Inst Med Trop São Paulo. 1979;21:266-8.

22. Maia MA, Campos DMB, Damasceno FA. Dipylidium caninum (Cestoda - Dilepididae). Relato de um caso humano em Goiania, Goiás. Rev Patol Trop. 1991;20(1):7-12.

23. Molina CP, Ogburn J, Adegboyega P. Infection by dipylidium caninum in an Infant. Arch Pathol Lab Med. 2004;127(3):157-9.
24. Coelho WAC, Sakamoto SM, Suassuna ACD, Ahid SMM, Assunção RHM. Larvas de ancilostomídeos em diferentes ambientes do Estado do Rio Grande do Norte. Rev Caatinga. 2007;20(3):80-2.

25. Nunes CM, Pene FC, Negrelli GB, Anjo CG, Nakano MM, Stobbe NS. Presence of larva migrans in sand boxes of public elementary schools, Araçatuba - Brazil. Rev Saúde Pub. 2000;34(6):656-8.

26. Santarém VA, Giuffrida R, Zanin GA. Larva migrans cutânea: ocorrência de casos humanos e identificação de larvas de Ancylostoma spp. em Parques públicos do município de Taciba, São Paulo. Rev Soc Med Trop. 2004;37(2):179-81.

27. Jackson A, Heukelbach J, Calheiros CML, Soares VL, Harms G, Feldmeier HA. A study in a community in Brazil in wich cutaneous larva migrans is endemic. Clin Infec Did. 2007;27(4):371-4.

28. Santos FAG, Yamamura MH, Vidotto O, Camargo PL. Ocorrência de parasites gastrintestinais em case (Canis familiaris) com diarréia agudo oriundos da região metropolitana de Londrina, estado do Paraná, Brasil. Semina: Ciências Agrárias. 2007;28(2):257-68.

29. Mendes-de-Almeida F, Silva MMO, Labarthe N. Giardia spp em amostras fecais de gatos domésticos do Rio de Janeiro, RJ. Acta Scient Vet. 2007; 35(2):468-9.

30. Monis PT, Andrews RH, Mayrhoffer G, Ey PL. Genetic diversity within the morphofogical species Giardia duodenalis and its relations to host origin. Infect, Gen and Evol. 2007;27(4):371-4.

31. Fok E, Szatmari V, Busak K, Rozgonyi F. Prevalence of intestinal parasites in dogs in some urban and rural areas of Hungria. Vet Quart. Utrecht. 2001;23(2):96-8.

32. Hackett T, Lappin MR. Prevalence of enteric pathogens in dogs of north-central Colorado. Am Anim Hosp Assoc. 2003;39(1):52-6. 
33. Trillo-Altamirano MP, Carrasco AJ, Cabrera R. Prevalência de helmintos enteroparásitos zoonóticos y factores associados em Canis familiaris em uma zona urbana de la cuidad de Ica, Peru. Parasitol Latinoamericana. 2003;58:136-41.

34. Crespo MV, Rosa F, Silva AE. Contaminação por parasitas em fezes de canídeos no concelho Peniche - dados preliminares. Acta Parasitol Port. 2006;13(1/2):53-7.

35. Fernandes BF, Roesel MP. Nascimento EE, Schultz JA. Considerações sobre parasitas gastrointestinais de cão (Canis familiaris) diagnosticados em exames de fezes. Arq Biol Tec. 1973;16:126-8.

36. Krychak S, Alcântara MA, Paula MC, Armstrong A, Gomes FB, Sypniewski D, et al. Levantamento epidemiológico de endoparasitas de case - Favela da Vila Torres - Parte I - Curitiba In: Anais XI Seminário Brasileiro de Parasitologia Veterinária, Salvador, SBPV; 1999. p. 173.

37. Tesseroli GL, Fayzano L, Agottani JVB. Ocorrência de parasitas gastrintestinais em fezes de cães e gatos, Curitiba, PR. Rev Acad. 2005;3(4):31-4.

38. Chieffi PP, Muller EE. Prevalência de parasitismo por Toxocara canis em cães e presença de ovos de Toxocara sp no solo de localidades públicas da zon urbana do município de Londrina, estado do Paraná Brasil Rev Saúde Pub. 1976;10:367-72.

39. Oliveira-Sequeira TCG, Amarante AFT, Ferrari TB, Nunes LC. Prevalence of intestinal parasites in dogs from São Paulo, State, Brazil. Vet Parasitol. 2002;103(1/2):19-27.

40. Silva AS, Ceolin LV, Cargnelutti JF, Pessoa GA, Oliveira CB, Quintal APN, et al. Prevalência de parasitismo em cães domiciliados num bairro de Santa Maria - RS. Saúde. 2007;33(1):27-31.

Recebido: 22/05/2007 Received: 05/22/2007

Aprovado: 05/08/2007

Approved: 08/05/2007 